\title{
Numerical Simulation and Analysis of Aerodynamic Characteristics of Road Vehicles in Platoon
}

\author{
Wei Gao ${ }^{1}$, Zhaowen Deng ${ }^{1}$, Ying Feng ${ }^{1}$, Yuping $\mathrm{He}^{2 *}$ \\ ${ }^{1}$ College of Automotive Engineering, Hubei University of Automotive Technology, Shiyan, China \\ ${ }^{2}$ Department of Automotive, Mechanical and Manufacturing Engineering, Ontario Tech University, Oshawa, Canada \\ *e-mail: yuping.he@uoit.ca
}

\begin{abstract}
With the spiking of fuel price and increasingly stringent emission regulation requirements, it brings a more daunting challenge for researchers and engineers to reduce the aerodynamic drag of road vehicles. When a vehicle is traveling in a platoon, the wake flow of the leading vehicle can affect the aerodynamic characteristics of the following vehicle. Due to the interaction of the flow field of the involved vehicles, the aerodynamic drag of each vehicle changes, which results in the alteration of the vehicle's fuel consumption. In the study, a single MIRA model was generated using CATIA software. The external flow field of the MIRA was imitated by CFD simulation. The numerical result of the drag coefficient was compared with the wind tunnel test results of Hunan University, China. The drag coefficient errors between the simulated value and the experimental result are less than $6 \%$. It implies that the simulation and tests achieve a good agreement. The benchmark indicates that the numerical simulation method is reliable. By means of CFD simulation, we explored the effects of separation distance, the number of vehicles in the platoon, the shape of the vehicle, and the speed of vehicle platoon on the aerodynamic properties of vehicles in platooning. The results of the numerical simulation demonstrate that although the influences of the aforementioned parameters on the aerodynamic properties of leading and trailing vehicles in the platoon are different, but the average drag coefficient of vehicle platoon is lower than that of a single vehicle, which is beneficial to improve the fuel economy of vehicle.
\end{abstract}

Keywords-automotive aerodynamics; drag reduction; platoon driving; CFD simulation; effect factors

\section{INTRODUCTION}

Recently, self-driving or autonomous vehicles have gained increasing attention in the automotive industry. There are many advantages for these vehicles in comparison with the human driver cars, such as improvement of safety, reduction of fuel consumption and $\mathrm{Co}_{2}$ emissions, increment of highway capacity, reduction of transportation costs, etc., and autonomous vehicles driving in platoon can further enhance the above advantages [1]. With the spiking of fuel price and increasingly stringent emission regulation requirements, it brings a more daunting challenge for researchers and engineers to reduce the aerodynamic drag of road vehicles.
Vehicle platooning is one of the ways to decrease the drag of vehicles. A lot of in-depth research was carried out regarding the platooning of general driver-controlled vehicles by simulations or experiments. The effect of the separation distance, the configuration of trailer, and lateral offsets on the drag reduction of two $1 / 15$ scale truck platooning were investigated using wind tunnel tests [2]. The results obtained from the study provide important guidelines for designing and developing autonomous vehicles. The aerodynamic characteristics of $1 / 20$ scale eight lorries platoon were experimentally studied by Robertson et al. It was found that the drag was affected by the separation distance of vehicles and the $57 \%$ drag reduction was obtained for the separation distance of a half of a vehicle length [3]. The fuel consumption and $\mathrm{CO}_{2}$ emission of automated truck platoons was researched using simulation and experiment. The results showed that the fuel consumption for the automated truck platoon was reduced by $14 \%$. The $\mathrm{Co}_{2}$ emission was decreased by $2.1 \%$ for a threetruck platoon at separate distance of $10 \mathrm{~m}$ [4]. The effect of various inter-vehicle spacing on the fuel consumption for two trucks platoon was investigated using CFD technique and wind tunnel test. The results indicated that the overall fuel economy of two truck platoon was improved as the separate distance decreased [5]. The aerodynamic characteristics and stability of the lorries platoon were investigated with delayed detached eddy simulations (DDES) and experiments [6]. Findings demonstrated that up to $60 \%$ reduction in drag was achieved for the intermediate trailing lorries. However, the lateral stability of lorries in the platoon was decreased due to platooning lorries resulting in strong vortex shedding. Lammert et al. studied the effects of the speed, separation distances, and gross vehicle weights on the fuel consumption of the tractor-trailer platoon [7]. The fuel consumption of the leading and trailing truck reduced by 5.3\% and 9.7\%, respectively. The flow field of the square-back Ahmed body and the simplified European truck in platoon was examined with numerical simulation [8]. The drag reduction of the threevehicle platoon was higher than the platooning of two vehicles. The maximum drag reduction of the trailing trucks was up to $40 \%$ for the three-vehicle platoon.

The aerodynamic properties of different platooning configurations in single and multiple lanes were investigated using CFD simulation method [9]. For two-vehicle platoon in a single lane, up to $23 \%$ of Yaw Averaged Drag (YAD) reduction and fuel saving were achieved. When the leading 
and trailing vehicles were on different lanes, the minimum team fuel savings were obtained at $30 \mathrm{ft}$ following distance. The team fuel economy of two and three trucks side-by-side on multiple lanes were reduced up to $11 \%$ and $29 \%$, respectively. The CFD simulation and track tests were performed to evaluate the fuel consumption of five-vehicle platoon [10]. The simulation results show that the drag coefficient was reduced by up to $20 \%$. The results of track tests demonstrate that the fuel-saving of trailing and leading vehicles was increased by $16 \%$ and $8 \%$, respectively. The influence of inter-vehicle spacing on the aerodynamic characteristics of two Ahmed bodies platoon was investigated through wind tunnel tests [11]. The drag of the trailing vehicle was increased significantly in comparison with the car in isolation when the separation distance is in the range of $\mathrm{x} / \mathrm{L}=0.1-1.0$, where $\mathrm{L}$ is the vehicle length. Furthermore, the lift coefficients of both vehicles platoon fluctuate greatly.

From the above review on the aerodynamic characteristics of vehicles in platoon, it is found that past studies were mainly focused on relatively short truck platoons due to the limitation of experimental and computational resources. Moreover, the main focus was on the effects of longitudinal spacing of vehicles in platoon on fuel consumption. However, the impacts of other parameters of vehicles in platoon, such as forward speed, number of vehicles, vehicle shape, etc., are less studied. In the current study, the impacts of the aforementioned parameters on the aerodynamic characteristics of passenger car platoons are explored using a Computational Fluid Dynamic (CFD) approach.

The rest of the paper is organized as follows. The simulation of the external flow field of single-vehicle and validation are presented in section II. Section III analyzes the effects on aerodynamic characteristics of vehicles in platoon due to the following factors: separation distance, number of vehicles, vehicle shape, and forward speed. The key conclusions are highlighted in section IV.

\section{SIMULATION AND VALIDATION OF EXTERNAL FLOW FIELD OF SINGLE-VEHICLE}

\section{A. Modeling}

The dimensions of the standard MIRA model group are shown in Fig.1 [12]. In the side view, the dashed lines denote oblique back and straight back model, respectively, while the respective solid line denotes ladder back model. Based on the dimensions shown in Fig.1, the 1/10 scale 3D MIRA models were generated using CATIA software, as shown in Fig. 2.

\section{B. Computational Domain and Meshing}

It is well-known that the proper size of the computational domain is crucially important for the accuracy of the numerical simulation results. To make the inlet flow uniform and stable, the wake flow fully developed, and avoid the generation of the backflow, the dimensions of the calculation domain are set as follows: the distance between the entrance of the computational domain and the front of the vehicle is 3 times vehicle length, the distance between the exit of the computational domain and the rear of the car body is 7 times vehicle length, the distance between the top surface of the computational domain and the upper surface of the car is 4 times vehicle height, and the distances between the side surfaces of the computational domain and the side surfaces of the car are 5 times vehicle width, respectively.

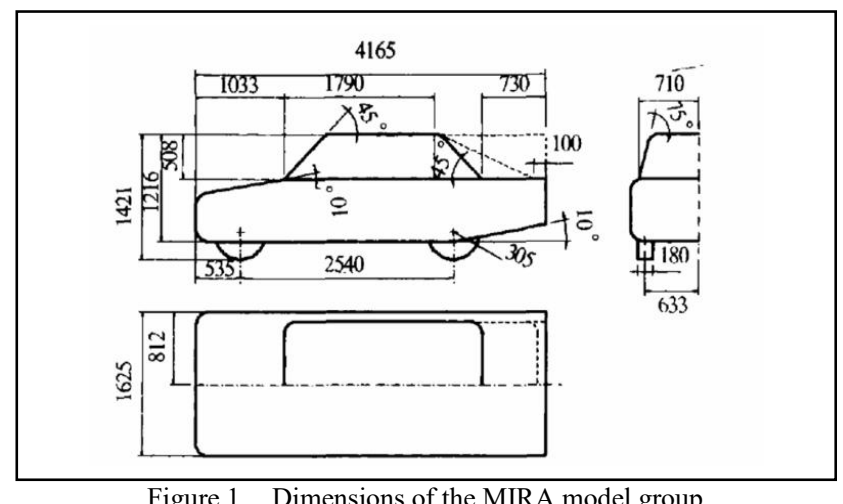

Figure 1. Dimensions of the MIRA model group

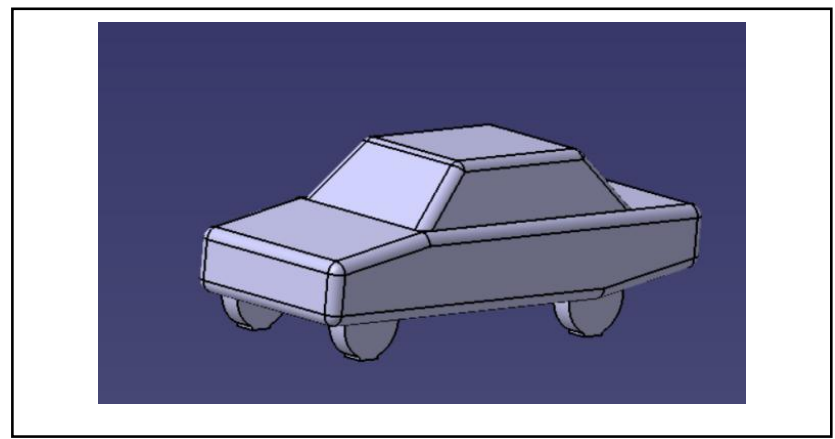

(a) Notchback

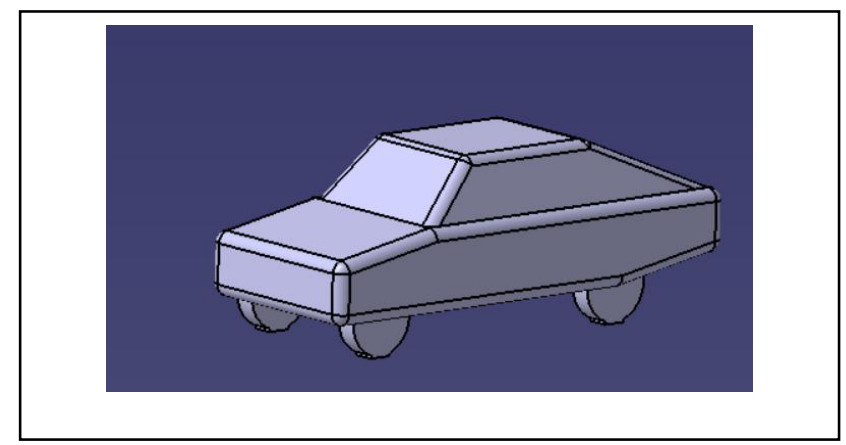

(b) Hatchback

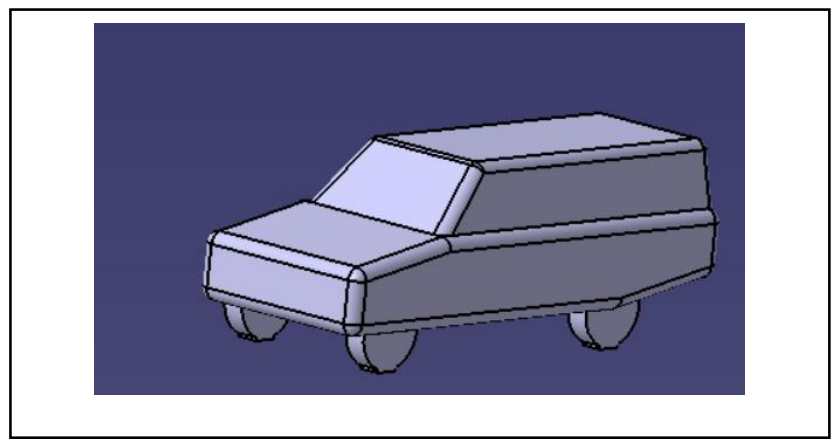

(c) Squareback

Figure 2. 3D MIRA model group 
The unstructured tetra meshes are generated using ANSYS software. To improve the accuracy of numerical simulation, the meshes around the vehicle are refined. Moreover, to obtain the effect of near-wall boundary on the flow and increase numerical convergence, the boundary layers of prism elements are added to the vehicle body surface.

\section{Boundary Conditions and Solver Settings}

The settings of the boundary conditions are particularly important for numerical simulation, which pose impacts on the convergence of the results of numerical calculation, and the error between simulation and experiment. The specific settings of the boundary condition are shown in TABLE I. The pressure-based solver is employed in the simulation. The realizable $k-\varepsilon$ turbulence model, standard wall function, second-order upwind solution method, simple scheme are used in the study. The residual value and the number of iterations are set to $10^{-6}$ and 2000 , respectively.

TABLE I. SETTINGS OF BOUNDARY CONDITIONS

\begin{tabular}{|c|c|}
\hline $\begin{array}{c}\text { Boundary of } \\
\text { Domain }\end{array}$ & Corresponding Conditions \\
\hline Inlet & $\begin{array}{c}\text { Velocity Inlet, Velocity Magnitude } \mathrm{V}=40 \mathrm{~m} / \mathrm{s}, \\
\text { Turbulent Intensity } \mathrm{I}=0.5 \% \text {, Turbulence Length } \\
\text { Scale } l=0.5 \mathrm{~m}\end{array}$ \\
\hline Outlet & $\begin{array}{c}\text { Pressure Outlet, Turbulent Intensity } \mathrm{I}=5 \%, \\
\text { Turbulence Length Scale } l=0.5 \mathrm{~m}\end{array}$ \\
\hline Ground & Moving wall, Speed U $=40 \mathrm{~m} / \mathrm{s}$ \\
\hline Other walls & Specified Shear \\
\hline $\begin{array}{c}\text { Vehicle body } \\
\text { surface }\end{array}$ & No-Slip \\
\hline
\end{tabular}

\section{Validation of Numerical Simulation}

To verify the CFD method, the simulation results are compared with the HD-2 wind tunnel test results achieved at Hunan University [13]. As shown in TABLE II, the errors are less than $6 \%$, which shows an excellent agreement between the simulation and test. Therefore, the numerical simulation method used in this paper is effective, which can be used for further investigation.

TABLE II. COMPARISON OF SIMULATION AND TEST OF DRAG COEFFICIENT

\begin{tabular}{|c|c|c|c|}
\hline Vehicle Type & Simulation & Wind Tunnel Test [13] & Error \\
\hline Notchback & 0.3156 & 0.3237 & $2.5 \%$ \\
\hline Hatchback & 0.2911 & 0.2749 & $5.8 \%$ \\
\hline Squareback & 0.3661 & 0.3870 & $5.4 \%$ \\
\hline
\end{tabular}

III. NUMERICAL ANALYSIS OF VARIOUS FACTORS ON AERODYNAMIC PERFORMANCE OF VEHICLES IN PLATOON

Vehicle platoons are also referred to as convoys, depicted in Fig. 3, have been recognized as an effective method to reduce the aerodynamic drag of road vehicles. In this section, the influences of nonuniform inter-vehicle spacings, number of vehicles, and vehicle shape on the aerodynamic performance of passenger car platoons are carried out using numerical simulation.

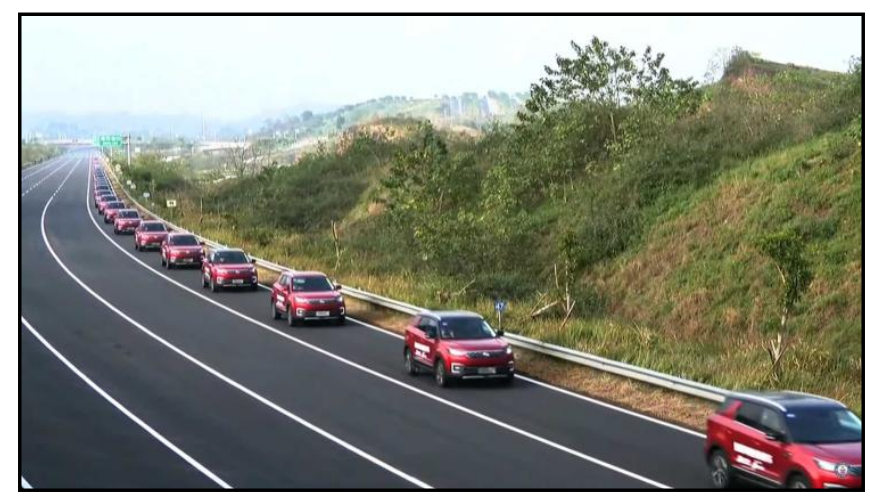

Figure 3. Schematic of vehicle platoon

\section{A. Effect of Longitudinal Spacing}

To study the influence of the longitudinal spacing on the aerodynamic characteristics of the vehicle platoon, the MIRA notchback model of two-vehicle platoon with different separation distances are generated using CATIA software. The numerical simulation of the two-vehicle platoon is conducted at the longitudinal spacing of $0.5 \mathrm{~L}, \mathrm{~L}, 1.5 \mathrm{~L}, 2 \mathrm{~L}, 2.5 \mathrm{~L}$, and $3 \mathrm{~L}$, respectively, where $\mathrm{L}$ is the vehicle length. The sizes of the computational domain are set as follows: the distance between the entrance of the computational domain and the front of the first vehicle is 3 times vehicle length, the distance between the exit of the computational domain and the rear of the last vehicle is 7 times vehicle length, other settings are the same as those for the single-vehicle cases. The setting of boundary conditions and solving parameters are consistent with the numerical simulation of the single-vehicle external flow field.

The average drag coefficient of the vehicles in the platoon is defined as follows [14]:

$$
C_{\text {Daverage }} / C_{D \infty}=\frac{1}{n} \sum_{i=1}^{\mathrm{n}} \frac{C_{D i}}{C_{D \infty}}
$$

where $\mathrm{C}_{\text {Daverage }}$ is the average drag coefficient of the platoon, $\mathrm{C}_{D i}$ is the drag coefficient of the ith vehicle in the platoon, $\mathrm{C}_{D \infty}$ is the drag coefficient of the corresponding single vehicle, $i$ is the ith vehicle in the platoon, and $n$ is the total number of vehicles in the platoon.

To evaluate the drag reduction characteristics of vehicle platoons, the average drag reduction rate of a vehicle platoon is defined as follows [15]:

$$
D_{R}=\frac{C_{D \infty}-C_{\text {Daverage }}}{C_{D \infty}}=1-\frac{1}{n} \sum_{i=1}^{n} \frac{C_{D i}}{C_{D \infty}}
$$

Fig. 4 shows the drag coefficient of the single, leading, trailing and the average drag coefficient of the vehicle platoon for various separation distances. For the leading vehicle, the drag coefficient first increases, then the rate of increase decreases in the range of $0.5 \mathrm{~L}-3 \mathrm{~L}$. The minimum drag coefficient is achieved at the separation distance of $0.5 \mathrm{~L}$. When the separation distance is greater than $1 \mathrm{~L}$, the drag coefficient of the leading vehicle is higher than that of the single-vehicle. 
In contrast, when the distance between the two cars increases from $0.5 \mathrm{~L}$ to $3 \mathrm{~L}$, the drag coefficient of the trailing vehicle is always less than that of the sing vehicle. At spacings over $2 \mathrm{~L}$, the drag coefficient of the rear vehicle changes slowly. The average drag coefficient of the vehicle platoon is lower than that of the single-vehicle within the range of $0.5-3 \mathrm{~L}$. When the separation distance is $0.5 \mathrm{~L}$, the average drag coefficient is the minimum.

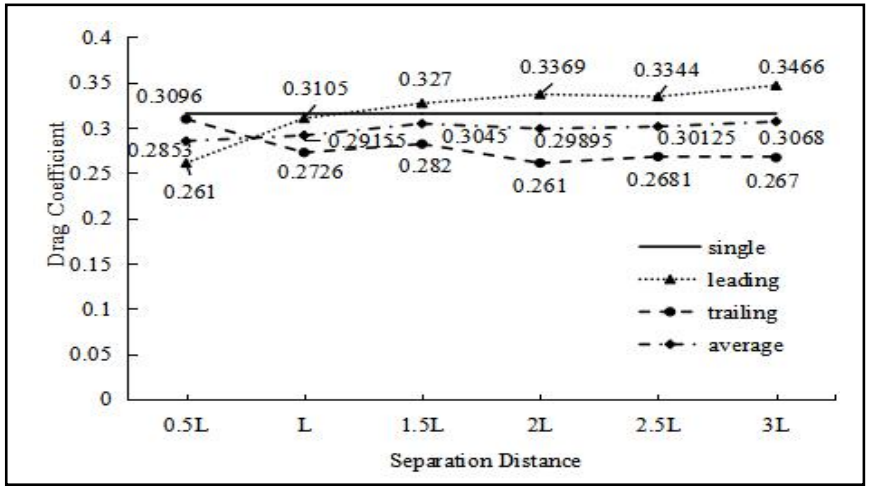

Figure 4. The drag coefficient of the vehicle platoon for various separation distances

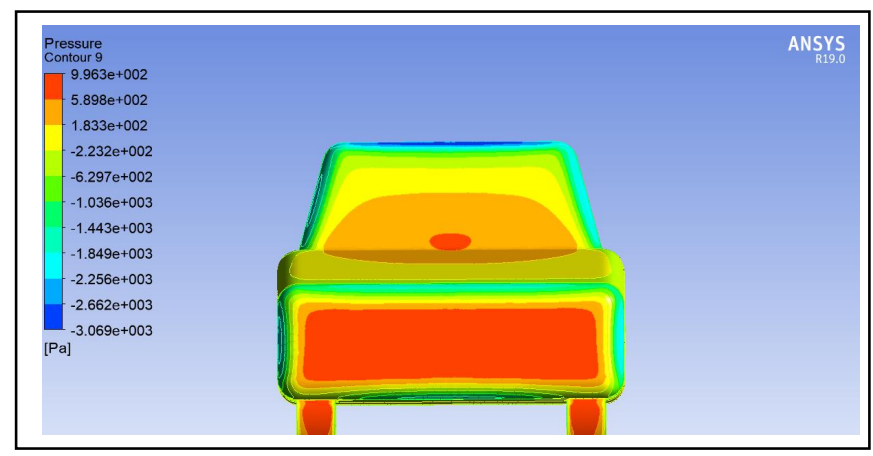

(a) single-vehicle

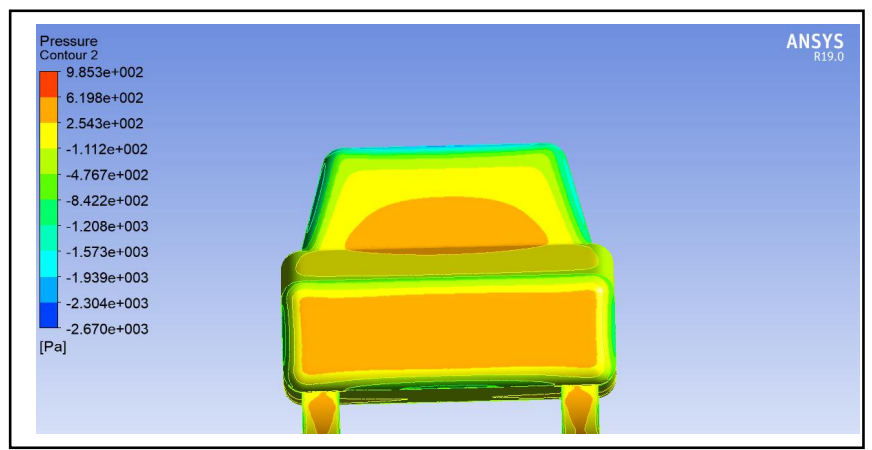

(b) trailing vehicle at the separation distance of $1 \mathrm{~L}$

Figure 5. Distribution of pressure at the front of the vehicles

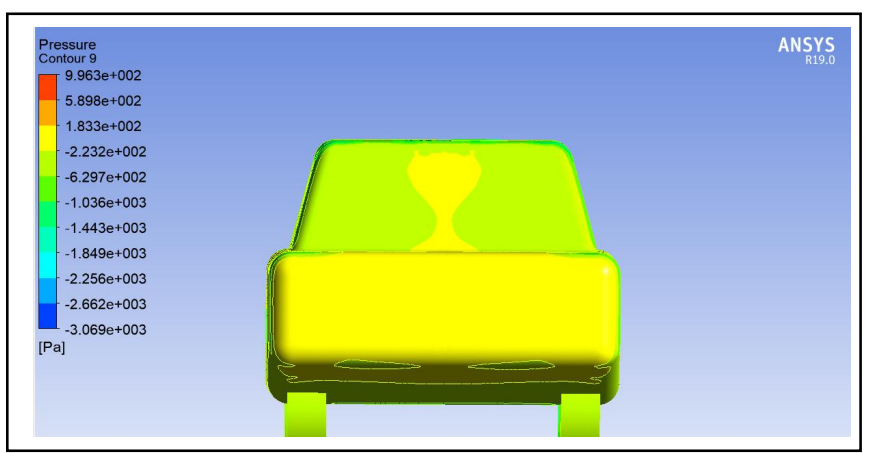

(a) single-vehicle

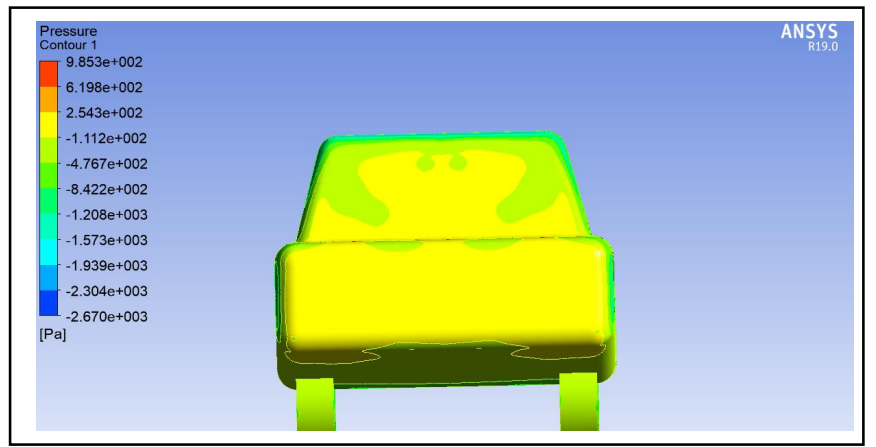

(b) leading vehicle at the separation distance of $1 \mathrm{~L}$

Figure 6. Distribution of pressure at the rear of the vehicle

When the vehicles are traveling in platoon, the trailing vehicle enters the wake region of the preceding car. Thus, the interaction effect of the vehicle platoon flow field occurs, which affects the aerodynamic properties of the vehicles in the platoon, especially the rear of the leading vehicle and the front of the trailing vehicle. The pressure distribution at the front of the single-vehicle and the trailing vehicle in the platoon are displayed in Fig. 5. The pressure at the front of the trailing vehicle with the separation distance of $1 \mathrm{~L}$ is lower than that of the single-vehicle. Therefore, the pressure difference between the front and the rear of the trailing vehicle in the platoon is decreased, which leads to the reduction of the drag coefficient. Fig. 6 shows the pressure distribution at the rear of the singlevehicle and the leading vehicle in the platoon. It discloses that the positive pressure region at the rear of the leading vehicle in the platoon is larger than that of the single vehicle, which causes the reduction of the pressure difference between the front and the rear of the leading vehicle at the separation distance of $1 \mathrm{~L}$. Thus, at the given separation, the drag coefficient of the leading vehicle in the platoon decreases compared against the drag coefficient of the single vehicle.

\section{B. Effect of the Numbers of Vehicles}

To examine the influence of the number of vehicles on the aerodynamic properties of vehicles in the platoon, the numerical simulation of multi-vehicle platoon is carried out using the MIRA notchback model at the longitudinal spacing of L. The number of vehicles in the platoon is $2,3,4,5,6$, and 7 , respectively. The settings of boundary conditions and solving parameters are consistent with those for the numerical simulation of the single-vehicle external flow field. TABLE III shows the drag coefficient of each vehicle in the platoon and 
the average drag coefficient of the platoon. It indicates that regardless of the variation of the vehicle number, the drag coefficient of the trailing car and the average drag coefficient of the vehicle platoon are less than that of the single-vehicle. When the numbers of vehicles in the platoon are more than three, the drag coefficient of the intermediate vehicle is less than that of the first and the last cars.

TABLE III. DRAG COEFFICIENT OF VEHICLES IN THE PLATOON

\begin{tabular}{|c|c|c|c|c|c|c|c|c|}
\hline Number & First & Second & Third & Fourth & Fifth & Sixth & Seventh & C $_{\text {Daverage }}$ \\
\hline two & 0.3105 & 0.2726 & & & & & & 0.2916 \\
\hline three & 0.3148 & 0.2489 & 0.2425 & & & & & 0.2687 \\
\hline four & 0.3214 & 0.2621 & 0.2451 & 0.2808 & & & & 0.2774 \\
\hline five & 0.3097 & 0.2508 & 0.2259 & 0.251 & 0.272 & & & 0.2619 \\
\hline six & 0.3207 & 0.2573 & 0.2403 & 0.2588 & 0.2693 & 0.2931 & & 0.2733 \\
\hline seven & 0.3037 & 0.2473 & 0.2352 & 0.2617 & 0.2701 & 0.2779 & 0.3021 & 0.2711 \\
\hline
\end{tabular}

The average drag reduction rate of the platoon with different number of vehicles is shown in Fig. 7. When the number of cars in the platoon increase from two to seven, the maximum average drag reduction rate is up to $17.0215 \%$ for the case with five. The average drag reduction rate is the minimum for the case of two. In addition, with the increase in the number of cars in the platoon, the amount of fluctuation is relatively smaller for the average drag reduction rate.

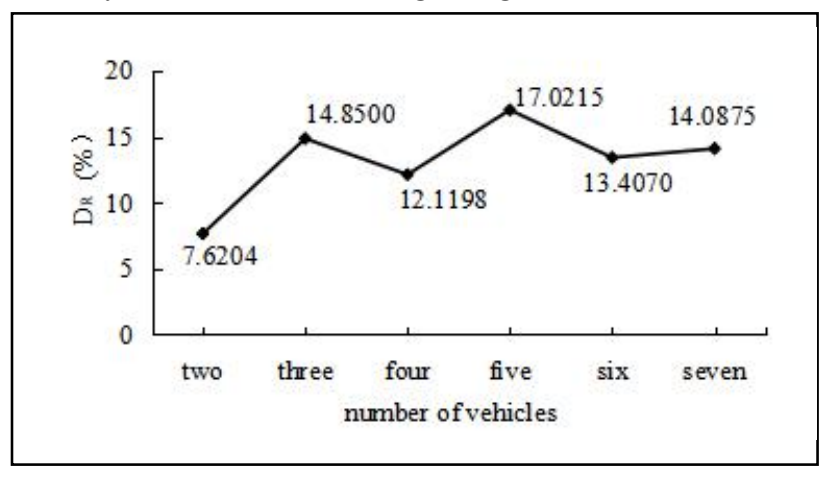

Figure 7. The average drag reduction rate of the platoon with different number of vehicles

\section{Effect of the Shape of Vehicles}

For high speed passenger vehicles, the pressure drag dominates about $90 \%$ of the total aerodynamic drag [16], and $91 \%$ of the pressure drag comes from the rear end of the vehicle [17]. Thus, the shape of a vehicle poses a great influence on the pressure drag. In this section, the influence of vehicle shapes on the aerodynamic properties of two vehicles in the platoon is evaluated. The simulation results of the drag coefficient are shown in TABLE IV. As shown in the table, the drag coefficient of the leading and trailing vehicle, the average drag coefficient of the two vehicles in the platoon is reduced in comparison with that of the single-vehicle.
TABLE IV. DRAG COEFFICIENT OF DIFFERENT SHAPE OF THE VEHICLE

\begin{tabular}{|c|c|c|c|c|}
\hline $\begin{array}{c}\text { Shape of } \\
\text { Vehicle }\end{array}$ & Leading & Trailing & C Daverage & Single Vehicle \\
\hline notchback & 0.3105 & 0.2726 & 0.2915 & 0.3156 \\
\hline hatchback & 0.2744 & 0.2839 & 0.2792 & 0.2911 \\
\hline suqareback & 0.3429 & 0.3300 & 0.3365 & 0.3661 \\
\hline
\end{tabular}

The relationship between the average drag reduction rate and the shape of the vehicle is illustrated in Fig. 8. It can be noticed that the platoon with the two MIRA squareback model has the highest average drag reduction rate and the value is up to $8.093 \%$. Although the drag coefficient of the single MIRA hatchback model is the minimum among the three models, the lowest average drag reduction rate is the platoon with the two MIRA hatchback model.

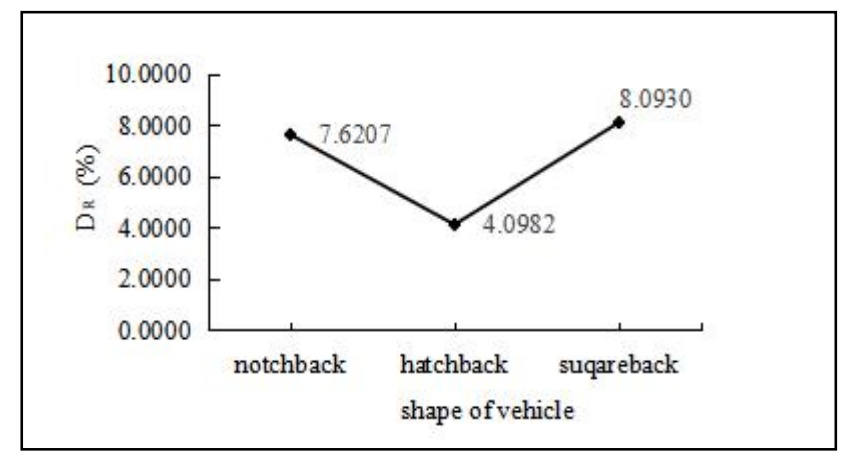

Figure 8. Average drag reduction rate for different shape of vehicle

\section{Effect of Vehicle Forward Speed}

It is reported that when vehicle speed reaches $80 \mathrm{~km} / \mathrm{h}$, the aerodynamic drag accounts for $50 \%$ of the driving resistance; as vehicle speed increases, the increased speed of aerodynamic drag is much faster than that of the rolling resistance [18]. It is necessary to assess the influence of vehicle forward speed on the aerodynamic characteristics of the vehicles in a platoon. As shown in TABLE V, for a two-vehicle platoon using the MRA notch back model, a total of 4 cases at four forward speeds are studied.

TABLE V. SIMULATION CASES FOR DIFFERENT FORWARD SPEEDS

\begin{tabular}{|c|c|c|}
\hline \multirow{2}{*}{ Vehicle Model } & Velocity of Vehicle & \multirow{2}{*}{$\begin{array}{c}\text { Separation } \\
\text { Distance }\end{array}$} \\
\hline \multirow{3}{*}{$\begin{array}{c}\text { MIRA notchback } \\
\text { model }\end{array}$} & $10 \mathrm{~m} / \mathrm{s}$ & \multirow{2}{*}{1} \\
\cline { 2 - 2 } $1 \mathrm{~L}$ \\
\cline { 2 - 2 } & $20 \mathrm{~m} / \mathrm{s}$ & \\
\cline { 2 - 2 } & $30 \mathrm{~m} / \mathrm{s}$ & \\
\hline
\end{tabular}

The drag coefficient of the single-vehicle, leading vehicle, trailing vehicle, and the average drag coefficient of the two-vehicle platoon at different speeds are shown in Fig. 9. The result seen in the figure indicates that the drag coefficients of both the leading and trailing vehicles in the platoon are lower than that of the respective single-vehicle at different speeds. The reduction of the drag coefficient for the trailing vehicle is larger than that for the leading vehicle. When the speed is $20 \mathrm{~m} / \mathrm{s}$, the average drag coefficient of the vehicle platoon reaches the minimal value. 


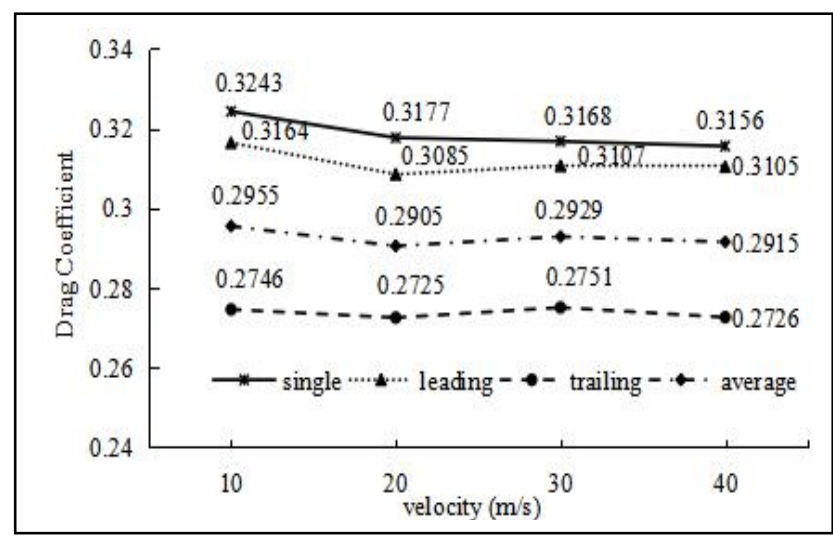

Figure 9. The drag coefficient of vehicle platoon for different velocity

\section{CONCLUSIONS}

In this paper, the external flow field of a single-vehicle has been simulated. The simulation results are validated using wind tunnel tests. Built upon this base, numerical simulations of the aerodynamic performance of vehicles in platoon are conducted using a CFD approach. The influences of separation distance, number of vehicles, vehicle shape, and forward speed on the aerodynamic properties of vehicles in platoon are analyzed. The main findings from the research are summarized as follows:

- When the separation distance of two vehicle in platoon is $0.5 \mathrm{~L}$ (where $\mathrm{L}$ is the length of the vehicle), the maximum of average drag reduction rate is $9.6 \%$. The average drag coefficient of the two vehicles in platoon is lower than that of the single-vehicle within the range of separation distance of $0.5-3 \mathrm{~L}$.

- Among the six platoons examined in the study, the average drag coefficient of the five-vehicle platoon is the minimum. The average drag coefficients for all the vehicle platoons are less than that of the single-vehicle.

- Among the three two-vehicle platoons with different MIRA models, the platoon with the MIRA notchback model shows the highest average drag reduction rate and its maximum value is up to $8.093 \%$, followed by the squareback and hatchback model.

- When the forward speed is in the range of $10 \sim 40 \mathrm{~m} / \mathrm{s}$, the drag coefficient of both the leading and trailing vehicles are lower than that of the single-vehicle. The average drag coefficient of the two-vehicle platoon with the MIRA notchback model achieves the minimum value at the speed of $20 \mathrm{~m} / \mathrm{s}$.

- When vehicles are traveling in platoon, the flow field of trailing vehicle is affected by that of leading vehicle, and vice versa. The aerodynamic properties of both the leading and trailing vehicles are influenced by the change of the wake region of the leading vehicle.

\section{ACKNOWLEDGMENT}

Financial support for this research by the Key Laboratory of Automotive Power Train and Electronics (Hubei University of Automotive Technology) [grant no. 2015XTZX0424], Auto
Parts Technology Hubei Province Collaborative Innovation Project [grant no. 2015XTZX04] are gratefully acknowledged.

\section{REFERENCES}

[1] Goldin, P., 2018. 10 advantages of autonomous vehicles. ITS Digest [Online]. Available from: inter-http://www.itsdigest.com/10-advantagesautonomous-vehicles.

[2] Brian R. McAuliffe, Mojtaba Ahmadi-Baloutaki, "A wind-tunnel investigation of the influence of separation distance, lateral stagger, and trailer configuration on the drag-reduction potential of a two-truck platoon," SAE Int. J. Commer. Veh., vol. 11, no. 2, pp. 125-150, 2018.

[3] Francis H. Robertson, Frederick Bourriez, Mingzhe He, et al. , "An experimental investigation of the aerodynamic flows created by lorries traveling in a long platoon," Journal of Wind Engineering \& Industrial Aerodynamics, vol. 193, article 103966, 2019.

[4] Sadayuki Tsugawa, Shin Kato, Keiji Aoki, "An automated truck platoon for energy saving," 2011 IEEE/RSJ International Conference on Intelligent Robots and Systems, September 25-30, 2011. San Francisco, CA, USA.

[5] Hugh Humphreys, David Bevly, "Computational fluid dynamic analysis of a generic 2 truck platoon," SAE Technical Paper, 2016-01-8008, 2016

[6] Mingzhe He, Shen Huo, Hassan Hemida, et al., "Detached eddy simulation of a closely running lorry platoon," Journal of Wind Engineering \& Industrial Aerodynamics, vol. 193, article 103956, 2019.

[7] Michael P. Lammert, Adam Duran, Jeremy Diez, et al., "Effect of platooning on fuel consumption of class 8 vehicles over a range of speeds, following distances, and mass," SAE Int. J. Commer. Veh., vol. 7, no. 2, pp. 626-639, 2014.

[8] Charles-Henri Bruneau, Khodor Khadra, Iraj Mortazavi, "Flow analysis of square-back simplified vehicles in platoon," International Journal of Heat and Fluid Flow, vol. 66, pp.43-59, 2017.

[9] Prasad Vegendla, Tanju Sofu, Rohit Saha, et al., "Investigation of aerodynamic influence on truck platooning," SAE Technical Paper, 2015-01-2895, 2015.

[10] Arturo Davila, Enric Aramburu, Alex Freixas, "Making the best out of aerodynamics: platoons," SAE Technical Paper, 2013-01-0767, 2013.

[11] Simon Watkins, Gioacchino Vino, "The effect of vehicle spacing on the aerodynamics of a representative car shape, "Journal of Wind Engineering and Industrial Aerodynamics, vol. 96 (6), pp.1232-1239, 2008 .

[12] Aerodynamic testing of road vehicles: Open throat wind tunnel adjustment, SAE J2071 Revised JUN94, 1994.

[13] Wang Shi, "Experiment Investigation on Aerodynamic Characteristics of MIRA Model Group in Wind Tunnel," Master Thesis, Hunan University, China, 2011.

[14] Fu Limin, Wu Yunzhu, He Baoqin, "Aerodynamic characteristics of vehicle platoon," Journal of Jilin University (Engineering and Technology Edition), vol. 36, no. 6, pp. 872-875, 2006.

[15] He Baoqin, Wu Yunzhu, Fu Limin, "Influence of vehicle shape on the aerodynamic characteristics of intelligent vehicle platoon," Journal of Jilin University (Engineering and Technology Edition), vol. 38, no. 1, pp. 7-11, 2008

[16] Kourta, A. and Gilliéron, P., "Impact of the automotive aerodynamic control on the economic issues," Journal of Applied Fluid Mechanics. vol. 2, no. 2, pp. $69-75,2009$.

[17] Ahmed S.R., Ramm R. and Faltin G., "Some salient features of the timeaveraged ground vehicle wake," SAE Transactions, vol. 93, 473-503. 1984.

[18] Junho cho, Tae-Kyung Kim, Kyu-Hong Kim,et al., "Comparative investigation on the aerodynamic effects of combined use of underbody drag reduction devices applied to real sedan," International Journal of Automotive Technology, vol. 18, no. 6, pp. 959-971, 2017. 